treatment had been completed. Many relatives can undertake simple observations, and in most areas the home nursing service can come into a house at the weekend to enable the patient to be looked after without undue risk. It seems that elderly patients are not adversely affected by a change of surroundings at the weekend and general practitioners do not find unreasonable demands made on them.

The reality of the situation is that when one walks round the wards in any general hospital at the weekend it is obvious that acceptance by society of a five-day week has affected the tempo of hospital work. There is very little in the way of routine investigations or use of the paramedical departments, and the nursing staff are often down to a minimum. Could not many general hospital patients be looked after at home at the weekend, and with regrouping certain wards be closed at the weekend? The nursing profession now relies on the married nurse to back up the hospital service, and the five-day ward is an attractive department for a nurse taking up work again when she has a family.

1 Naylor, R., The Lennard Hospital. Unpublished.

3 Home for the Weekend-Back on Monday. A report sponsored by the King Edward's Hospital Fund. London, Queen's Institute of District Nursing, 1973.

\section{Effects of Cholecystectomy}

Surprisingly little is known about the effect of cholecystectomy on alimentary function-perhaps because until recently there has been only limited understanding of the role of the gallbladder in gastrointestinal physiology other than its function in storing and concentrating hepatic bile. The gallbladder is absent in cetacea and certain ungulates and rodents; nevertheless these mammals do not appear to be at any digestive disadvantage. It is tempting to speculate that the presence of a gallbladder confers some advantage by delivering a small volume of a concentrated bile-salt solution at the time of ingestion of large quantities of animal fat. If this is so, how does the person without a gallbladder deal with the fat in the diet? As far as can be ascertained, very well indeed. Simmons and Bouchier ${ }^{1}$ studied the intraluminal bile-salt concentrations after a test meal in 10 subjects who had undergone cholecystectomy some 3 months to 12 years previously. Fat digestion seemed unimpaired. Whereas persons with a normally functioning gallbladder show a rise and then a fall in intraluminal bile-salt concentrations in the two hours after a meal, no such phenomenon is found in the absence of a gallbladder, when the concentration of bile salts in the gut remains constant throughout the two hour period. The bile-salt concentration is usually greater than the $4 \mathrm{mmol}$ which Badley et al..$^{2}$ have suggested is the critical physiological concentration necessary for the emulsification and absorption of a normal fat load. Even if bile-salt concentrations do on occasions fall below this concentration in patients who have had a cholecystectomy it is improbable that fat digestion and absorption will be affected adversely. Porter et $a l .{ }^{3}$ showed that even patients with external biliary fistulae absorb most of the dietary fatty acids; apparently luminal hydrolysis of triglycerides can proceed effectively in the absence of bile salts, and there is a large reserve of functioning intestinal mucosa for the absorption of the fatty acids. Cholecystectomy is not followed by steatorrhoea, and none of the symptoms that may occur after gallbladder surgery have ever been shown to be due to the absence of the gallbladder.

Recently it has become apparent that cholecystectomy is accompanied by changes in bile-salt metabolism, which indicates that there may be a subtle and complex relation between normal gallbladder function and the secretion of bile salts. The first indication that this might be so was the observation that cholecystectomy is accompanied by a change in biliary lipid chemistry. ${ }^{56}$ Hepatic bile, previously satur ated or supersaturated with cholesterol, becomes unsaturated. This suggests that the gallbladder might influence bile salt kinetics, and support for this concept comes from the observation of Low-Beer and Pomare ${ }^{7}$ that patients with untreated coeliac disease have a two-to-threefold increase in the size of the taurocholate pool with a doubling of the half life of this bile salt. They suggested that this is due to the poor emptying of the gallbladder and retarded enterohepatic circulation of bile salts known to occur in adult coeliac disease. ${ }^{8}$

Two studies have evaluated the effect of cholecystectomy on bile-salt metabolism in greater detail. Almond et al. ${ }^{9}$ studied biliary lipid composition and the size of the bile-salt pool before and after cholecystectomy in 10 patients. In contrast to previous studies ${ }^{5} 6$ they found no change in lipid composition, and the bile remained saturated with cholesterol after removal of the gallbladder. After cholecystectomy five of the patients showed a small expansion of the primary bile-salt pool, while in the remaining five there was a further reduction of the size of the bile-salt pool, already noted to be smaller than usual before operation. The fractional turnover rates of cholate and chenodeoxycholate were increased, suggesting that in the absence of the gallbladder there is an increased frequency of enterohepatic cycles. There was no change in the amount of secondary bile salts. In the second study Pomare and Heaton ${ }^{10}$ evaluated 13 patients after cholecystectomy and compared the results with 10 matched controls. They found reduced pools of the primary bile salts with deoxycholate, a secondary bile salt, becoming the predominant component; and they argue that the removal of the gallbladder is accompanied by a reduction in pool size, which they attribute to an increased number of enterohepatic circulations causing a continuous feedback inhibition of hepatic bile-salt synthesis. Pomare and Heaton believe that these results support their hypothesis that the storage function of the gallbladder is an important determinant of the size of the bile-salt pool and that the pool size is determined by the frequency of the enterohepatic circulation of the bile salts. This is in contrast to the conclusion of Almond et al. that the gallbladder does not have an important role in influencing bile chemistry or the size of the bile-salt pool. Northfield and Hofmann ${ }^{11}$ have suggested that an increased contractility of the gallbladder might cause increased recycling of the bile salts thereby inducing more marked feedback inhibition of bile-salt synthesis and consequently a reduced bile-salt pool size.

So arguments have been put forward that a smaller bile-salt pool might result from a primary defect in liver metabolism, or might be a reflection of a failure of gallbladder filling or an absence of the gallbladder, or might be due to enhanced gallbladder contraction. Almost certainly multiple factors can affect pool size: and their varying effects in different circumstances no doubt explain the apparently conflicting results obtained by different investigators.

\footnotetext{
1 Simmons, F., and Bouchier, I. A. D., South African Medical fournal, 1972, 46, 2089.

2 Badley, B. W. D., Murphy, G. M., and Bouchier, I. A. D., Lancet, 1969 2,400 .

Porter, H. P., Saunders, D. R., Tytgat, G., Brunser, O., and Rubin, C. E., Gastroenterology, 1971, 60, 1008.
} 
4 Krondl, A., Vavrinkova, H., and Michalec, C., Gut, 1964, 5, 607.

5 Simmons, F., Ross, A. P. J., and Bouchier, I. A. D., Gastroenterology, $1972,63,466$

6 Shaffer, E. A., Braasch, J. W., and Small, D. M., New England Fournal of Medicine, 1972, 287, 1317.

7 Low-Beer, T. S., and Pomare, E. W., British Medical Fournal, 1973, 2, 338.

${ }^{8}$ Low-Beer, T. S., Heaton, K. W., Heaton, S. T., and Read, A. E., Lancet, 1971, 2, 991.

${ }^{9}$ Almond, H. R., Vlahcevic, Z. R., Bell, C. C. Jr., Gregory, D. H., and Swell, L., New England fournal of Medicine, 1973, 289, 1213.

10 Pomare, E. W. and Heaton, K. W., Gut, 1973, 14, 753.

11 Northfield, T. C., and Hofmann, A. F., Lancet, 1973, 1, 747.

\section{Brenner Tumours}

One of the rarer neoplasms of the ovary is the tumour first identified in 1907 by Brenner. ${ }^{1}$ Typically it is solid, resembling a fibroma in appearance, and its size varies from a microscopical lesion to a mass weighing over $8 \mathrm{~kg} .^{2}$ Microscopically the tumour consists of round or cylindrical nests of epithelial cells invested in a dense fibrocellular stroma. The epithelial cells-strikingly uniform in appearance-are large and polyhedral, and their prominent nuclei often show a characteristic longitudinal grooving reminiscent of a coffee-bean. Many of the nests have a tubular or cystic structure, and the central cavity may contain rounded protoplasmic masses superficially resembling ova. In some of these cystic spaces the superficial layer of Brenner cells may be replaced by a mucus-secreting columnar epithelium. On occasions there may be large cystic cavities lined by this mucinous epithelium to an extent that the tumour becomes indistinguishable from the common mucinous cystadenoma. It has indeed been argued that the Brenner tumour represents extensive squamous metaplasia in a mucinous cystadenoma, ${ }^{3}$ but it is much more likely that the mucinous change develops in a pre-existing Brenner tumour, since it is scanty in small tumours and most extensive in large ones. Furthermore, when mucus-secreting epithelium is present in the typical nests showing cavitation it is found centrally, which suggests that it was formed more recently than the Brenner cells.

The histogenesis of the tumour is uncertain. Brenner believed it was of follicular origin because of the ovum-like structures frequently seen in the cavities of the cell nests, ${ }^{1}$ but very few workers now accept this theory. ${ }^{4}$ Meyer ${ }^{5}$ drew attention to the similarity of the cell nests to the congenital inclusions sometimes found in the ovary called Walthard's rests; but these are also present in the Fallopian tubes and uterine ligaments, sites in which the Brenner tumour has not been described. One current view is that the tumour arises from the ovarian mesothelium (also called coelomic epithelium and germinal epithelium) which grows down in a branching fashion into the ovary and subsequently undergoes squamous and later mucoid metaplasia. Indeed some Walthard rests might develop in a similar way. The other view is that the tumour is derived from the mesonephric remnants in the ovary, the rete ovarii.

Clinically Brenner tumours usually occur in postmenopausal women. They are hormonally inert in the great majority of cases, but occasionally there is evidence of oestrogenic activity with endometrial hyperplasia. Even more rarely there may be androgenic effects. These uncommon endocrine manifestations are probably due to a transformation of the stromal cells adjacent to the epithelial nests into cells capable of producing steroids. ${ }^{2}$

Brenner tumours were originally believed to be invariably benign, but more recently an increasing number of malignant examples have been reported. In some instances the mucussecreting epithelium undergoes malignant change, when the tumour falls into the category of mucinous cystadenocarcinoma, a common type of ovarian cancer. It is, however, rarer to find authentic examples of carcinomatous change in the Brenner epithelium. ${ }^{6}$ Recently Hull and Campbell ${ }^{7}$ reported an incontrovertible case of malignant Brenner tumour occurring in a 52-year-old woman who died of multiple metastases three years after hysterectomy and bilateral oophorectomy. Histologically the tumour showed a gamut of changes extending from typically benign microcystic islets and areas of proliferating transitional epithelium to foci of anaplastic transitional-cell transformation. No mucus-secreting epithelium was present. The authors stress the strict criteria that must be applied before a malignant Brenner tumour is diagnosed. The tumour should be an anaplastic or transitional-cell carcinoma of the ovary, intimately associated with a benign Brenner tumour, and preferably showing epithelial transition. If mucinous elements are present they should be well separated from the areas of epithelial transition between the benign and malignant parts of the tumour. There should be evidence of stromal invasion, which is manifested by a gross attenuation of the stroma by extremely large epithelial masses, for cytological appearances alone are inadequate in the diagnosis of an invasive cancer. Finally there should be no evidence of a primary carcinoma in another site.

1 Brenner, F., Frankfurt Zeitschrift für Pathologie, 1907, 1, 150.

Novak, E. R., and Woodruff, J. D., Novak's Gynecologic and Obstetric Pathology, 6th edn., p. 379. Philadelphia, Saunders, 1967.

3 Woodruff, J. D., and Acosta, A. A., American Fournal of Obstetrics and Gynecology, 1962, 83, 657

4 Teoh, T. B., Fournal of Pathology and Bacteriology, 1953, 66, 441.

5 Meyer, R., Archiv für Gynäkologie, 1932, 148, 541 .

6 Idelson, M. G., Obstetrical and Gynecologic Survey, 1963, 18, 246.

7 Hull, M. G. R., and Campbell, G. R., Obstetrics and Gynecology, 1973, $42,527$.

\section{Do We Know What We Are Doing?}

Surgeons are immediately distinguishable from physicians by their extrovert attitudes and behaviour-it is in their nature to be doers rather than observers. One consequence of this difference in temperament, at least according to an American view, ${ }^{1}$ is that surgeons are less self-critical. They and their patients tend to believe that an operation must be effective, and certainly better than a pill or no treatment at all. This attitude is reflected in the papers they write, and indeed having reviewed 49 articles on cardiac surgery in 16 journals $D . H$. Spodick ${ }^{1}$ found that not one satisfied strict criteria of a scientifically designed study.

Do these conclusions apply to British medicine? The controlled clinical trial can be claimed as a British innovation. Moreover, in general the standard of assessment of the results of any form of treatment is probably higher today than 20 years ago. This owes something to high standards of refereeing by journals, education through specialist societies, and the scientific co-operation of some pharmaceutical firms. Nevertheless, a quick glance through any journal, particularly a surgical one, reveals far too many articles of the "I did this and this is what happened" variety. Such papers are sometimes necessary if they draw attention to an important unexpected occurrence, but they are worthless as part of the scientific assessment of treatment and probably do harm by publishing unsubstantiated information. 